\title{
Relative importance of adaptation and genotype $\times$ environment interactions in tropical beef breeding systems
}

\author{
Heather Burrow ${ }^{\dagger}$ \\ Cooperative Research Centre for Beef Genetic Technologies, CJ Hawkins Homestead, UNE, 2350 Armidale, Australia
}

\section{Introduction}

Cattle grazed in (sub) tropical environments are subjected to numerous stressors such as parasites, seasonally poor nutrition, high heat and humidity and endemic diseases transmitted by parasites. The impact of each stressor on production and animal welfare is often multiplicative rather than additive, particularly when animals are already undergoing physiological stress (e.g. lactation). Under the extensive production systems common in the (sub) tropics, it is generally not possible to control the stressors through management strategies alone. The best method of reducing the impacts of these stressors so to improve productivity and animal welfare is to breed cattle that are productive in their presence, without the need for managerial interventions.

Maximising beef production and profitability requires the genotype be matched to the specific production environment. Some genotypes are better suited to particular environments, with no one breed being 'best' in all environments. Genotype $\times$ environment $(G \times E)$ interactions may impact significantly on productivity across different environments. They are indicated by (i) re-ranking of breeds or sires within breeds for performance across different environments; or (ii) genetic correlations between environments that are antagonistic or, if positive, less than unity (e.g. $<0.80$ ). Recently, interest has focused on possible changes in Single Nucleotide Polymorphism (SNP) associations with economically important traits across environments as a third indicator of $\mathrm{G} \times \mathrm{E}$. This review examines the importance of adaptation and the extent of $\mathrm{G} \times \mathrm{E}$ in (sub) tropical beef production systems to identify opportunities to simultaneously improve productivity and cattle adaptation.

\section{Breed types and their adaptation to (sub) tropical environments}

For most purposes in the (sub) tropics, breeds can be categorized into general groupings (Bos taurus; Bos indicus; tropically adapted taurine breeds; and Bos indicus-derived composites; Burrow et al., 2001). In temperate areas there may be substantial differences in performance between these breed types. But in the (sub) tropics, differences in performance are masked by the effects of environmental stressors on productive attributes (e.g. growth and fertility). In the presence of stressors, productive attributes of poorly adapted cattle are significantly reduced relative to their performance in temperate environments or relative to the performance of adapted cattle in the (sub) tropics.

\section{Re-ranking of breeds across environments}

Burrow et al. (2001) and Burrow (2006) cite many studies indicating differences between breeds that are significant and large for most economically important traits. However the ranking of the breeds is not always consistent across environments. Re-ranking of breeds across environments is best managed through use of breed types most suited to the particular production environment.

\section{Re-ranking of sires within breeds}

Re-ranking of sires within breeds across environments potentially has a greater impact on beef production than re-ranking of breeds because it is easier and less expensive to change to a better-adapted breed than to identify sires within breeds that are genetically superior for the full range of productive and adaptive traits required in the tropics. From studies in Australia, USA, Canada and Uruguay, it appears that G $\times E$ in Bos taurus breeds may have a significant impact across extreme tropical and temperate environments or where animals are selected in stressfree environments for use in (sub) tropical environments. However, $\mathrm{G} \times \mathrm{E}$ interactions are not of major importance for Bos taurus breeds across the temperate environments where they are traditionally grazed. There is little evidence of significant re-ranking of tropically adapted sires for a wide range of productive traits when reared in tropical or temperate environments. This supports evidence from selection experiments that, in tropically adapted breeds, adaptive traits are genetically independent of productive traits (Burrow, 2001). G $\times E$ interactions may provide new opportunities to differentially select to simultaneously improve performance of tropically adapted steers in benign environments and their half-sib heifers for use in tropical environments.

\section{Re-ranking of SNP}

Unpublished data from Australia, USA and Canada indicate significantly greater discord between significant SNP for growth, feed efficiency and beef quality attributes in Bos taurus steers between Australia and North America relative to USA and Canada (Bennett G.L., Goddard, M.E.,

\footnotetext{
†E-mail: Heather.Burrow@une.edu.au
} 
Miller, S.P., Thallman, R.M. et al., unpublished results). These results support published results from laboratory species (Fry et al., 1998) and broiler chickens (Long et al., 2008) across different environments. Hence GxE interactions will be important considerations in SNP association analyses of complex beef production traits in future.

\author{
References \\ Burrow HM 2001. Livestock Production Science 70, 213-233. \\ Burrow HM 2006. Proceedings $8^{\text {th }}$ World Congress Genetics Applied to Livestock Production ISBN 85-60088-01-6. \\ Burrow HM, Moore SS, Johnston DJ, Barendse W and Bindon BM 2001. Australian Journal of Experimental Agriculture 41, 893-919. \\ Fry JD, Nuzhdin SV, Pasyukova EG and Mackay TFC 1998. Genetical Research, Cambridge 71, 133-141. \\ Long N, Gianola D, Rosa GJM, Weigel A and Avendaño S 2008. Journal of Animal Science 86, 3358-3366.
}

\title{
Genotype by environment interaction on growth and carcass traits in beef cattle in the tropics
}

\author{
Fabrizio Assenza ${ }^{1 *}$, Alberto Menendez Buxadera ${ }^{1}$, Jean-Luc Gourdine ${ }^{1}$, Alain Farant ${ }^{2}$, \\ Bruno Bocage ${ }^{2}$, Xavier Godard ${ }^{2}$ and Michel Naves ${ }^{1+}$
}

'INRA, UR143, Unité de Recherches Zootechniques, 97170 Petit Bourg, Guadeloupe, France; ${ }^{2}$ INRA, UE1294, Plateforme Tropicale d'Experimentation sur I'Animal, 97170 Petit Bourg, Guadeloupe, France; *Animal breeding and genetics Group, P.O. Box 3386700 AH, Wageningen, The Netherlands

\section{Introduction}

A wide variety of beef cattle production systems may be found in the tropics, which could be classified as pasture based systems, mixed farming systems, and industrial systems, following the classification by Seré and Steinfeld (1995). In tropical regions, pasture based systems and mixed faming systems are mainly practiced, with different levels of intensification in management. In that context, one major goal for cattle breeders is to match the type of animal they use to the management conditions they apply.

In this study, our objective was to investigate the genotype $\times$ environment interaction (GEI) that may affect growth traits (live weight, average daily gain, yearling weight) and carcass traits (slaughter weight, hot carcass weight, muscular tissue weight, adipose tissue weight and digestive tract weight) expressed across two contrasted systems.

\section{Material and methods}

The analysis was made in a database of 718 young Creole calves, born between 1999 and 2008, and distributed between two management conditions during their post weaning growth. The calves were born in different suckling herds, and weaned at about 211 days, at a weaning weight of $157 \mathrm{~kg}$, on average. After weaning and an adaptation period of 40 days on average, 346 steers and 372 heifers were separated into two groups, each on a different management system. The penned animals ( 176 steers and 200 heifers) were fattened indoor, and fed with cut grass and concentrate (maize: $68 \%$; wheat middling: $22 \%$; soya meal: $8 \%$; UF $=1.05$; PDIN $=113 \mathrm{~g}$ ), distributed up to $60 \%$ of their voluntary intake. The grazed animals (196 steers and 146 heifers), were fed outdoor at pastures, only with grazed tropical forages. During the entire experiment, the animals were regularly weighted every 2 weeks. At the end of the growing period, 298 steers were sacrificed. Slaughter ages were defined in order to obtain equivalent live weight range at the end of the fattening period: 14-17 months for penned steers, and 17-21 months for grazing steers. As soon as they arrived at the slaughter house, the steers were kept fasting for 24 hours, and then weighted just before slaughter. The calves were issued from 32 sires and 163 dams; the pedigree was 6 generations deep and a total of 961 animals are included in the pedigree file. Descriptive statistics were obtained with the $\mathrm{SAS}^{\odot}$ software, and the genetic analysis was performed with the ASREML and MATLAB software. The longitudinal dataset collecting the observations on live weight of each animal at different ages was analyzed by a random regression with Legendre polynomials of order two fitted for age, animal effect and permanent individual effect. The fixed part of the model included a fixed effect coding for contemporary groups (by sex, management system, group of weighting), and the covariables age and weight at weaning; the random part included the genetic animal effect and the permanent environment individual effect. The datasets collecting observations on the other traits were analyzed by several bivariate models. The genetic correlations between traits expressed within the same environment were computed by implementing bivariate models where the response variables were two different traits, expressed within the same environment. The GEI affecting each trait was measured by implementing

\footnotetext{
† E-mail: michel.naves@antilles.inra.fr
} 